\title{
Design of electron band pass filters for electrically biased finite superlattices
}

\author{
J. Martorell \\ Departament d'Estructura i Constituents de la Materia, Facultat Física, University of Barcelona, Barcelona 08028, Spain \\ D. W. L. Sprung and G. V. Morozov \\ Department of Physics and Astronomy, McMaster University, Hamilton, Ontario, Canada L8S 4M1
}

(Received 18 September 2003; published 11 March 2004)

\begin{abstract}
We design optimal band pass filters for electrons in semiconductor heterostructures, under a uniform applied electric field. The inner cells are chosen to provide a desired transmission window. The outer cells are then designed to transform purely incoming or outgoing waves into Bloch states of the inner cells. The transfer matrix is interpreted as a conformal mapping in the complex plane, which allows us to write constraints on the outer cell parameters, from which physically useful values can be obtained.
\end{abstract}

DOI: 10.1103/PhysRevB.69.115309

PACS number(s): 73.21.Ac, 73.21.Cd, 73.23.Ad

\section{INTRODUCTION}

In previous work we have studied how to modify a finite periodic superlattice with a few additional cells so as to obtain optimal electron transmission for most of the energies of a specific allowed miniband. ${ }^{1-3}$ The resulting device is a band pass filter and the additional cells are called antireflection coatings (ARC's), in analogy to optics. Improving on the work of Pacher et al., ${ }^{4,5}$ we showed in Ref. 1 that very simple devices consisting of a small number of identical elements $(N \geqslant 5)$ sandwiched between two optimally designed ARC cells could achieve transmissivities as high as $80 \%$. In the present work we extend the methods of Ref. 1 to design band pass filters operating under the bias of a constant electric field. As is well known, such a field tends to strongly suppress coherent electron transmission across a superlattice at most miniband energies. Recently, however, electron transport via the Wannier-Stark resonances of four- and fiveperiod finite superlattices has been experimentally observed and characterized by the Vienna group. ${ }^{6}$ These experiments (and those in Refs. 4 and 5) hint that with similar devices it should be possible to detect the coherent transmission across band pass filters like those considered here.

The study of ARC's was proposed ${ }^{4}$ as a novel means of arriving at better designs for the injectors of quantum cascade lasers. ${ }^{7}$ In addition it should provide complementary information on the transition from coherent to incoherent transport in finite superlattices ${ }^{8}$ : whereas for a finite superlattice without ARC the transmission is dominated by resonant transport, we will show that with ARC cells transport proceeds by a combination of Bloch states and resonances. Since the Bloch states transmit directly across the lattice, it is expected that decoherence will be less likely, due to reduced transit time, in such devices.

Other theoretical work on the design of band pass filters for electrons in the absence of electric bias exists. Gaylord and collaborators ${ }^{9-11}$ studied the problem, adapting methods from optics and microwaves. Chang and $\mathrm{Kuo}^{12}$ took a similar conceptual approach using the language of impedance transformers. Tung and Lee, ${ }^{13,14}$ as well as Gomez et al., ${ }^{15}$ considered filters based on a Gaussian distribution of barrier strengths. Simanjuntak and Pereyra ${ }^{16}$ have explored resonant transmission in finite periodic systems. But to our knowledge, work on filters under electric bias is scarce. The first designs were proposed by Glytsis et al. ${ }^{10}$ These were based on semiclassical ideas which apply to above-barrier transmission, whereas the experiments and designs in Ref. 4 sought to improve transmission in the first and second minibands, both well below the barrier energy.

In Sec. II we describe the transfer matrix properties required for a fully quantal approach to filter design. Section III describes our method of determining the device parameters, while in Sec. IV we give results for examples close to the experimental conditions for AlGaAs-GaAs devices.

\section{TRANSFER MATRICES}

We work in the envelope function approximation; the stationary-state wave function for the electron is obtained as a solution of the one-dimensional Schrödinger equation with an effective mass. ${ }^{17}$ We denote the potential inside the device as $V(x), x_{L}<x<x_{R}$, and assume it to be constant outside. To allow for the action of a nonvanishing electric field inside, these constant values are taken to be different: $V_{L}, V_{R}$. We write the outside electron wave functions at energy $E$ as

$$
\begin{aligned}
& \Phi_{L}(x)=\frac{a_{L}}{\sqrt{\nu_{L}}} e^{i k_{L}\left(x-x_{L}\right)}+\frac{b_{L}}{\sqrt{\nu_{L}}} e^{-i k_{L}\left(x-x_{L}\right)}, \quad x \leqslant x_{L}, \\
& \Phi_{R}(x)=\frac{a_{R}}{\sqrt{\nu_{R}}} e^{i k_{R}\left(x-x_{R}\right)}+\frac{b_{R}}{\sqrt{\nu_{R}}} e^{-i k_{R}\left(x-x_{R}\right)}, \quad x \geqslant x_{R},
\end{aligned}
$$

where

$$
k_{L, R} \equiv \sqrt{2 m_{L, R}^{*}\left(E-V_{L, R}\right) / \hbar^{2}},
$$

and $\nu_{L, R}=\hbar k_{L, R} / m_{L, R}^{*}$ is the velocity. The transfer matrix is defined to relate the coefficients

$$
\left(\begin{array}{l}
a_{L} \\
b_{L}
\end{array}\right)=M\left(\begin{array}{l}
a_{R} \\
b_{R}
\end{array}\right) .
$$


Hermiticity of the Hamiltonian and flux conservation lead to $\operatorname{det} M=1$ and $M_{22}=M_{11}^{*}, M_{21}=M_{12}^{*}$. Thus, at any energy $E$, only three real quantities are required to specify $M$. Also, these relations lead to the representation for $M$ in terms of transmission and reflection amplitudes

$$
M=\left(\begin{array}{cc}
1 / t_{L} & r_{L}^{*} / t_{L}^{*} \\
r_{L} / t_{L} & 1 / t_{L}^{*}
\end{array}\right) .
$$

For an incoming wave from the left $b_{R}=0$ and then $t_{L}$ $=a_{R} / a_{L}$ and $r_{L}=b_{L} / a_{L}$. Finally, the transmission and reflection probabilities are

$$
T=\left|t_{L}\right|^{2} \quad \text { and } \quad R=\left|r_{L}\right|^{2} .
$$

Our devices are made of $N$ consecutive pieces, hereafter called cells. The total transfer matrix is the product of the transfer matrices of those cells, which we number from left to right:

$$
M=M_{1} M_{2} \cdots M_{N} .
$$

Guided by experiments on electron transport in GaAsAlGaAs superlattices, we consider here devices made from uniform layers of two materials (called well and barrier material). The cells are chosen so as to have reflection symmetry by placing two half-well layers of the same thickness, $w / 2$, on either side of the barrier of thickness $b$ and height $V_{b}$ relative to the well. That makes the potential symmetric in the absence of external field. We call them square barrier cells, though this applies only in zero applied field. The method employed can be applied to any shape potential cell and is not limited to bilayer devices. Reflection symmetry is not essential, but reduces the number of independent parameters describing the transfer matrix and simplifies the design; it was used in our previous work. ${ }^{1-3}$ In the presence of an external constant field the potential is still piecewise linear, but reflection symmetry is destroyed. The explicit construction of the transfer matrix, under the bias of a constant electric field, is explained in detail in the Appendix. These single-cell transfer matrices have the same properties given above for a general matrix $M$, so the following discussion applies equally to the total transfer matrix of the device or to any component.

It is convenient to take the trace of $M$ as one of the three real quantities which specify it. According to the above relations,

$$
\operatorname{Re}\left(M_{11}\right)=\frac{1}{2} \operatorname{Tr} M \equiv \cos \phi .
$$

Values of the energy for which $\left|\operatorname{Re}\left(M_{11}\right)\right| \leqslant 1$ (real angles $\phi$ ) define the allowed bands of an infinite periodic system. Forbidden zones correspond to the remaining values of $E$. The other two parameters will be related to the fixed points of a conformal mapping, as described in the next subsection.

\section{Conformal mapping in the complex plane}

To choose the remaining two real quantities, we note ${ }^{18,19}$ that $M$ allows one to define a mapping in the complex plane, $w(z)$, as follows: the amplitudes left and right of the cell are related by Eq. (2) and therefore, defining $z \equiv b_{R} / a_{R}$ and $w$ $\equiv b_{L} / a_{L}$, one has

$$
w(z)=\frac{M_{21}+M_{22} z}{M_{11}+M_{12} z} .
$$

This mapping is known as the bilinear or Möbius mapping and is a conformal mapping of the entire plane onto itself and which maps circles into circles.

Properties of this mapping are part of any course in complex variables and have recently been discussed, in the present context, in Refs. 18-20. Here we summarize those whose physical interpretation is relevant for the present work.

(i) The flux of a state written as in Eq. (1), with components $(a, b)$, is $j=|a|^{2}-|b|^{2}$ and is conserved by the action of $M$. Therefore, waves with net flux to the right, $j>0$, correspond to points $|z|<1$ which are mapped onto points $|w|$ $<1$. Reciprocally wave functions with net flux to the left, $j<0$, correspond to $|z|>1$ and are mapped onto $|w|>1$. The unit circle also maps into itself. According to Eq. (3), the image of $z=0$ is $w(0)=M_{21} / M_{11}=r_{L}$, the reflection amplitude. The condition for perfect transmission is $w(0)=0$ : the origin mapped onto the origin. In terms of the transfer matrix,

$$
M\left(\begin{array}{c}
a_{R} \\
0
\end{array}\right)=\left(\begin{array}{c}
a_{L} \\
0
\end{array}\right)
$$

with $\left|a_{R}\right|=\left|a_{L}\right|$.

(ii) For an infinite periodic system made of identical cells, a Bloch wave is a state such that

$$
M\left(\begin{array}{c}
a_{ \pm} \\
b_{ \pm}
\end{array}\right)=e^{ \pm i \phi}\left(\begin{array}{c}
a_{ \pm} \\
b_{ \pm}
\end{array}\right),
$$

where $\phi$ is the Bloch phase: $\cos \phi=\operatorname{Re}\left(M_{11}\right)$. It then follows that the ratios $b_{ \pm} / a_{ \pm}$are precisely the fixed points of the mapping. When $\phi$ is real (allowed band), these two states propagate freely in the periodic system. The fixed points satisfy $w\left(z_{f}\right)=z_{f}$ which leads to

$$
M_{12} z_{f}^{2}+2 i \operatorname{Im} M_{11} z_{f}-M_{21}=0,
$$

with solutions

$$
z_{f}=\frac{1}{M_{12}}\left\{-i \operatorname{Im}\left(M_{11}\right) \pm \sqrt{\left[\operatorname{Re}\left(M_{11}\right)\right]^{2}-1}\right\},
$$

where we have used $\operatorname{det} M=\left|M_{11}\right|^{2}-\left|M_{12}\right|^{2}=1$. In a forbidden band the square root is a real number and it is immediate that $\left|z_{f}\right|=1$, so that the two fixed points are on the unit circle. In an allowed band the square root is imaginary and

$$
z_{f}=\left[-\operatorname{Im}\left(M_{11}\right) \pm \sin \phi\right] \frac{e^{i \chi}}{\left|M_{12}\right|} .
$$

(The phase of $M_{12}$ is written as $\pi / 2-\chi$.) Of the two Bloch states one describes propagation with net flux to the left and 
the other to the right. Therefore, one of the two fixed points lies inside the unit circle, $z_{f, \text { in }}$, and the other outside, $z_{f, \text { out }}$; both have the same phase $\chi$.

Equation (11) also shows that when the energy is varied towards a band boundary, the fixed points approach the unit circle. When $\left|\operatorname{Re}\left(M_{11}\right)\right|=1$ the two fixed points reach the unit circle at the same point $e^{i \chi}$. For energies in a forbidden band the fixed points travel in opposite directions around the unit circle and they join again at the boundary of the next allowed band. We will later show examples of this behavior, which is also discussed in Ref. 20.

The above analysis suggests that we choose the remaining two real quantities defining $M$ as $\left|z_{f, i n}\right|$ and $\chi$. Thus, for an energy in the allowed band, $M$ is completely determined once the fixed point and the Bloch phase $\phi$ are specified. In particular, for a symmetric potential cell, $M_{12}$ is pure imaginary and then Eq. (12) shows that in an allowed band the fixed points lie on the real axis, with $\chi=0$. Then only two real parameters are needed.

Although the above properties hold at a fixed energy, we have found ${ }^{1-3}$ that over most of an allowed band, the position of the fixed point changes very little, while $\phi$ changes by $\pi$. In this case, as the energy changes, the mapping moves the system point on an arc of a circle enclosing the fixed point. When device properties are optimized near the middle of an allowed band, the performance persists over a range of energies on either side of the Bragg energy.

(iii) In terms of the mapping, the ARC design proposed by us in Ref. 1 can be obtained as follows. Consider a device made of $N$ identical and symmetric cells, with transfer matrices $U$, clad left and right by single ARC cells with transfer matrices $A^{\prime}$ and $A$, so that the total transfer matrix is $M$ $=A^{\prime} U^{N} A$. This corresponds to a band pass filter operating at zero electric field. The parameters of the $N$ inner cells are dictated by the desired energy window for the filter. The potential in each cell is chosen so that the desired transmission window corresponds to the lowest allowed band of $U$. To determine the profiles of the ARC cells, we note that the corresponding mapping is of the form

$$
w(z)=w_{A^{\prime}}\left(w_{U}\left(\cdots w_{U}\left(w_{A}(z)\right) \cdots\right)\right),
$$

where $w_{U}$ acts $N$ times. To achieve perfect transmission $w(0)=0$, one must design the ARC cells so that they first transform purely outgoing waves into Bloch states of $U$, the first step of the above mapping, and these into purely ingoing waves, the last step of the mapping.

Therefore we construct $A$ so that $w_{A}(0)=z_{f, \text { in }}$

$$
\frac{A_{21}}{A_{11}}=z_{f, i n} .
$$

The last step $w_{A^{\prime}}\left(z_{f}\right)=0$ requires that

$$
A_{21}^{\prime}+A_{22}^{\prime} z_{f, i n}=0 .
$$

The simplest solution uses a symmetric cell for $A$. Using the properties of symmetric potentials (real fixed points and $A_{21}^{*}=-A_{21}$ ), one can show that Eq. (15) follows from Eq. (14), so that the same ARC cell serves both purposes: $A^{\prime}$
$=A$. By writing out the matrix elements of $A$ and $U$ explicitly one sees easily that Eq. (14) is the same as Eq. (A3) in Ref. 1 . We showed there that imposing condition (14) at an energy near the middle of the allowed band of $U$ allows one to determine the parameters of ARC cells, and with these one obtains a transmission coefficient close to unity over most of the lowest allowed band.

\section{ARC DESIGN FOR NONZERO ELECTRIC BIAS}

We now apply the mapping technique to the design of band pass filters operating under a constant electric field $F$. The devices will be made by assembling individual square barrier cells as above. Following the previous example we divide the design into two stages: (i) find the individual parameters of each of the inner $N$ cells so as to have a desired optimal transmission window and (ii) construct the single cell ARC cladding.

\section{A. Band alignment of the inner cells}

Due to the bias, the central part of the potential now has a nonsymmetric shape, and for a given incident energy, each cell has a different transfer matrix $M^{(i)}, i=1, \ldots, N$. The total transfer matrix for this central part will be denoted $M_{c}$ and the corresponding mapping by $w_{c}(z)$. In order to have a sharp cutoff of transmission at the edges of the pass band, one wants the transfer matrices of the cells forming this inner part to have (nearly) the same allowed band boundaries: $E_{b}, E_{t}$. However, the action of an applied field with potential $e F x$ shifts the energies of the band boundaries of a cell by approximately $e F d_{c}$, where $d_{c}$ is the distance from the center of the cell to the origin. Therefore, an inner part of identical cells will not work. To compensate for the shift one has to choose different parameters $w, b$, and $V_{b}$, for each square barrier cell, and require that they produce the desired band boundaries, $\operatorname{Re}\left[M_{11}^{(i)}\left(E_{b, t}\right)\right]= \pm 1, i=1, \ldots, N$, when the external field is applied. However, since we wish to optimize transmission in the middle of the energy range, and not at the boundaries, we prefer to impose one alignment at the Bragg point energy $E_{B}$ instead. The Bragg point is defined by the condition $\cos \phi\left(E_{B}\right)=0$ and lies near the middle of an allowed band. In conclusion, the conditions used to determine the inner cell parameters are

$$
\begin{gathered}
\operatorname{Re}\left[M_{11}^{(i)}\left(E_{B}\right)\right]=0, \\
\operatorname{Re}\left[M_{11}^{(i)}\left(E_{t}\right)\right]=-1,
\end{gathered}
$$

and, for each $F$, allow one to compute $w^{(i)}, b^{(i)}$, and $V_{b}^{(i)}$.

\section{B. ARC cladding}

Once the $M^{(i)}$ have been determined, their product gives $M_{c}$ and the corresponding fixed point of $w_{c}(z), z_{f, i n}$. To have perfect transmission at the chosen energy, Eqs. (14) and (15) still apply, but because the system is no longer symmetric, we cannot conclude that the upstream and downstream ARC cells are the same, and indeed they are not. We will show in the applications in the following sections how Eqs. 
(14) and (15) allow one to compute the parameters of the two ARC cells under a given bias, $F$.

\section{RESULTS}

To show how this method can be applied, we will discuss devices with $N=3$ inner cells and single-cell ARC claddings. (See the inset to Fig. 7 for an illustration.) For a narrow barrier, the three-layer square barrier cell (for instance, GaAs-AlGaAs-GaAs used in experiments) can be well approximated by shrinking the barrier to zero width, becoming a $\delta$ function at the center, $V(x)=C \delta\left(x-x_{b}\right)$. This simple potential profile has the advantage of fewer (two) parameters: $\delta$ strength, $\Omega \equiv m^{*} C / \hbar^{2}$, and cell width $d$, which simplifies the solution of the above equations. We shall first discuss this didactic case and afterwards present results for more realistic square barrier cell devices. For simplicity, most of the numerical calculations have taken a constant effective mass across all cells of the device.

\section{A. Design and results for $\delta$ cell filters}

A number of exact results and accurate approximations are available for $\delta$ cells.

(i) When $F=0$, the upper band boundary is exactly at

$$
E_{t}=\frac{\hbar^{2}}{2 m^{*}}\left(\frac{\pi}{d}\right)^{2},
$$

whereas, in the limit of $\Omega d \gg 1$ (Ref. 20),

$$
E_{B} \simeq \frac{\hbar^{2}}{2 m^{*}}\left(\frac{\pi}{d}\right)^{2}\left(1+\frac{1}{\Omega d}\right)^{-2} .
$$

(ii) For a cell with center at $x_{b}$, it can be proved that, to lowest order in $e F$,

$$
\begin{gathered}
E_{t}(F)=E_{t}(0)+e F x_{b}, \\
E_{B}(F)=E_{B}(0)+e F x_{b} .
\end{gathered}
$$

We have chosen the zero of potential energy due to the electric field at the middle point of the middle cell. To align the bands sufficiently, we proceed as follows: First we choose the middle cell parameters, subindex $m$, so as to have the desired allowed band boundaries at $F=0$. This can be easily done by using Eq. (17) of Ref. 1. The parameters of the left cell are next determined so that

$$
\frac{\hbar^{2}}{2 m^{*}}\left(\frac{\pi}{d_{l}}\right)^{2}-e F \frac{d_{m}+d_{l}}{2}=\frac{\hbar^{2}}{2 m^{*}}\left(\frac{\pi}{d_{m}}\right)^{2},
$$

which determines $d_{l}$, while $\Omega_{l}$ follows from

$$
\begin{gathered}
\frac{\hbar^{2}}{2 m^{*}}\left(\frac{\pi}{d_{l}}\right)^{2}\left(1+\frac{1}{\Omega_{l} d_{l}}\right)^{-2}-e F \frac{d_{m}+d_{l}}{2} \\
=\frac{\hbar^{2}}{2 m^{*}}\left(\frac{\pi}{d_{m}}\right)^{2}\left(1+\frac{1}{\Omega_{m} d_{m}}\right)^{-2} .
\end{gathered}
$$

The corresponding expressions allow one to determine $d_{r}$ and $\Omega_{r}$. The first example that we will analyze is the one
TABLE I. $\delta$ cell filters with middle cell $\Omega_{m}=1.88 \mathrm{~nm}^{-1}, d_{m}$ $=5.55 \mathrm{~nm}$. $\delta$ cell parameters for various electric fields, $F$.

\begin{tabular}{lccc}
\hline \hline$e F(\mathrm{meV} / \mathrm{nm})$ & Cell & $\Omega\left(\mathrm{nm}^{-1}\right)$ & $d(\mathrm{~nm})$ \\
\hline-1.0 & $a^{\prime}$ & 0.5839 & 4.8955 \\
& $l$ & 1.7753 & 5.6424 \\
& $r$ & 1.9810 & 5.4634 \\
& $a$ & 0.6294 & 4.5343 \\
\hline \multirow{2}{*}{3.0} & $a^{\prime}$ & 0.5262 & 5.3405 \\
& $l$ & 1.5695 & 5.8476 \\
& $r$ & 2.1873 & 5.3051 \\
& $a$ & 0.6623 & 4.2232 \\
\hline \multirow{2}{*}{5.0} & $a^{\prime}$ & 0.4515 & 5.9136 \\
& $l$ & 1.3632 & 6.0876 \\
& $r$ & 2.3943 & 5.1633 \\
& $a$ & 0.6806 & 3.9384 \\
\hline \hline
\end{tabular}

already studied in Ref. 1 for $F=0$. The middle cell parameters are $d_{m}=5.55 \mathrm{~nm}$ and $\Omega_{m}=1.88 \mathrm{~nm}^{-1}$, while $m^{*} / m_{e}$ $=0.071$. We then determine the left and right inner cell parameters as described above. The results are shown in Table I for several choices of $F$. The resulting $\cos \phi$ for the three aligned cells are plotted in Fig. 1 as a function of $k$ $\equiv \sqrt{2 m^{*} E / \hbar^{2}}$, and as can be seen, the band boundaries are well aligned. Also shown is the real part of the total $M_{c, 11}$ matrix element $\left(M_{c} \equiv M_{l} M_{m} M_{r}\right)$. Note that if the three inner cells were identical, as when $F=0$, then ${ }^{21} \operatorname{Re}\left(M_{c, 11}\right)$ $=\cos (3 \phi), \phi$ being the Bloch angle of a single cell, and the corresponding curve would be very similar to the solid line in Fig. 1, but would be tangent to the dotted horizontal lines at \pm 1 . With nonvanishing electric field we find that the tangent points near $k \simeq 0.50 \mathrm{~nm}^{-1}$ and $0.54 \mathrm{~nm}^{-1}$ develop into narrow forbidden zones. To facilitate discussion we denote the boundaries of the three allowed zones of $\operatorname{Re}\left(M_{c, 11}\right)$ as

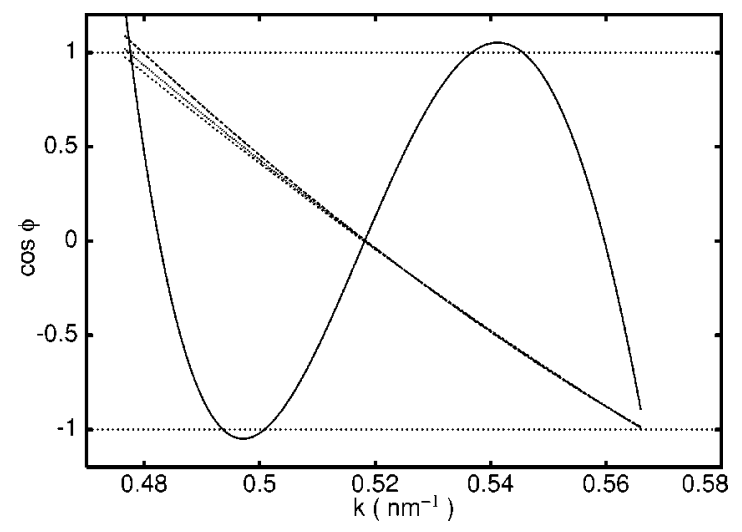

FIG. 1. Bloch phases for the $\delta$ cell filter of Table I with $e F=$ $-5 \mathrm{meV} / \mathrm{nm} . \cos \phi$ for left (long dashed line), middle (dotted), and right (short dashed) cells. The solid line is $\operatorname{Re}\left(M_{c, 11}\right)$. 


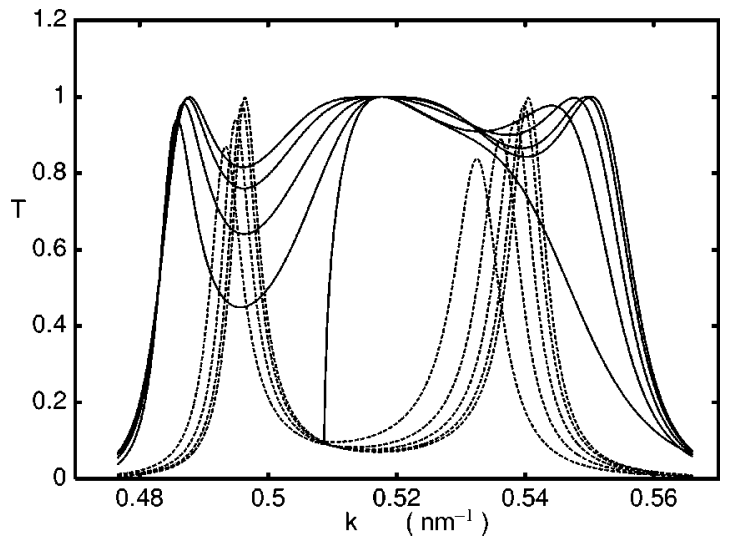

FIG. 2. $T$ for the $\delta$ cell filters of Table I, with (solid line) and without ARC (dotted line.) $e F=-1,-3,-5$, and $-9 \mathrm{meV} / \mathrm{nm}$.

$\left(b^{\prime}, t^{\prime}\right),\left(b^{\prime \prime}, t^{\prime \prime}\right)$, and $\left(b^{\prime \prime \prime}, t^{\prime \prime \prime}\right)$.

In the case of $N$ identical inner cells, it is known ${ }^{21}$ that for the energies leading to $\cos N \phi= \pm 1$, resonant transmission occurs with $T=1$. We show in Fig. 2 that similar peaks occur when $F \neq 0$, but that the development of forbidden zones reduces the peak transmission. Our calculations indicate that the wave functions at the energies of these resonant peaks are related to the symmetric and antisymmetric quasibound states supported by a three-barrier structure.

To construct the ARC cells, we use Eqs. (14) and (15), choosing an energy near the middle of the allowed band-for convenience, at the Bragg energy. Each of Eqs. (14) and (15) gives two real equations to determine two real parameters. In practice, to find $\Omega_{a}$ and $d_{a}$ for the rightmost cell, we minimize $\left|A_{21} / A_{11}-z_{f, \text { in }}\right|$ using a simplex subroutine from $\mathrm{Nu}-$ merical Recipes. By this means, we have always found solutions that exactly fulfill Eq. (14). Similarly we find the $\Omega_{a}$, and $d_{a^{\prime}}$ for the leftmost ARC cell. The resulting values are included in Table I.

Figure 2 shows the transmission for filters with and without ARC's. Without ARC's one sees two narrow peaks located near the energies of the forbidden zones of Fig. 1. After adding the ARC, a third flat peak appears at $k=k_{B}$. This central peak is always rather wide, but the two resonant peaks remain, although shifted towards the band boundaries. The net result is a rather satisfactory filter for most values of $F$, with abrupt jumps from $T=0$ to 1 and a rather flat top covering most of the chosen allowed band. At the highest electric field shown, there is a sharp drop in the transmission curve, with $\mathrm{ARC}$, at $k_{\text {min }} \simeq 0.51 \mathrm{~nm}^{-1}$. This value corresponds closely to the condition that the incoming electron energy, $E=\hbar^{2} k^{2} /\left(2 m^{*}\right)$, must exceed the potential energy at the left of the device:

$$
V_{L}=|e F|\left(d_{a^{\prime}}+d_{l}+\frac{1}{2} d_{m}\right)=\frac{\hbar^{2} k_{m i n}^{2}}{2 m^{*}}
$$

For positive values of $e F$ a similar condition holds; the electron must have enough energy to overcome the potential step at the right, $V_{R}$.

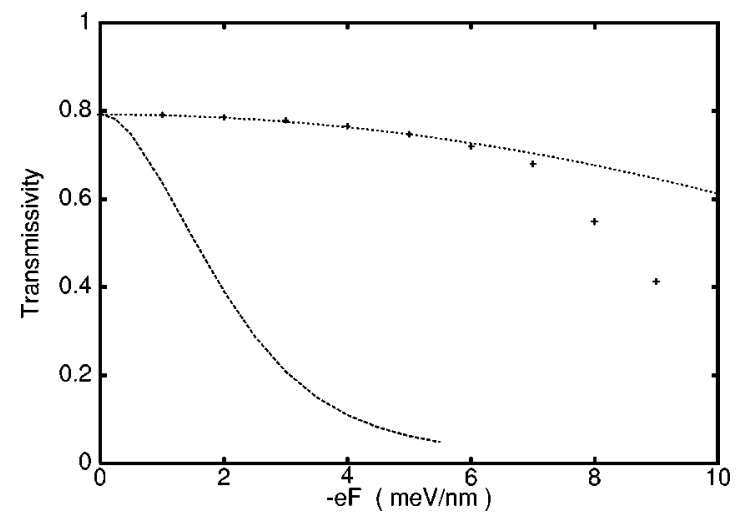

FIG. 3. Symbols + : transmissivities for the $\delta$ cell filters of Table I, at the electric fields for which they are optimal. The dotted line is a parabolic fit that shows the trend at moderate $F$ 's. The dashed line is the transmissivity for the filter optimized at zero electric field.

$$
V_{R}=|e F|\left(d_{a}+d_{r}+\frac{1}{2} d_{m}\right) \leqslant \frac{\hbar^{2} \widetilde{k}_{\text {min }}^{2}}{2 m^{*}} .
$$

These conditions will also be relevant for the example discussed in the next subsection.

We defined the average transmissivity in Eq. (13) of Ref. 1:

$$
\tau=\frac{1}{E_{t}-E_{b}} \int_{E_{b}}^{E_{t}} T(E) d E .
$$

In Fig. 3 points marked by + show values of $\tau$ for the filters of Table I at the electric field for which they have been optimized. Up to $e F \simeq-7 \mathrm{meV} / \mathrm{nm}$, the transmissivity of these filters exceeds $70 \%$. For comparison we also show the transmissivity computed at different electric fields, for the filter that was optimal at zero electric field. As can be seen the decrease in $\tau$ is much faster for the filter with fixed parameters.

Returning to the results in Fig. 2, depending on the incident energy $E$, transmission proceeds through two kinds of states: (i) Bloch-like states in the middle part of the transmission window and (ii) resonant states like those already present without the ARC's, at either end of the energy band. To focus on the ARC behavior, in Fig. 4 we show the trajectory of the fixed point, $z_{f, i n}(E)$, when the energy varies from the bottom $b^{\prime}$ to the top $t^{\prime \prime \prime}$ of the allowed bands of the single cells. Corresponding to the three allowed bands of the composite cell, $M_{c}$, shown in Fig. 1, the fixed point describes three arcs inside the unit circle. Starting at $E=E_{b^{\prime}}$, the fixed point is on the unit circle (point $b^{\prime}$ in the figure). As the energy increases the fixed point moves clockwise along the dotted line path up to $E=E_{t^{\prime}}$ (point $t^{\prime}$ ). There it rejoins the unit circle which it follows until the energy reaches the bottom of the next allowed band of $M_{c}$, point $b^{\prime \prime}$ in the figure. Next from $E_{b^{\prime \prime}}$ to $E_{t^{\prime \prime}}$ it follows the continuous line path to point $t^{\prime \prime}$. It travels again on the unit circle as $E$ increases towards $E_{b^{\prime \prime \prime}}$ and finally describes a second dotted path from $b^{\prime \prime \prime}$ to $t^{\prime \prime \prime}$ when $E$ increases to the upper allowed band edge at $E_{t^{\prime \prime \prime}}$. On these paths, the stars correspond to increases in momentum, $k$, of $5 \%$ of the difference $k_{t^{\prime \prime \prime}}-k_{b^{\prime}}$. As can be 


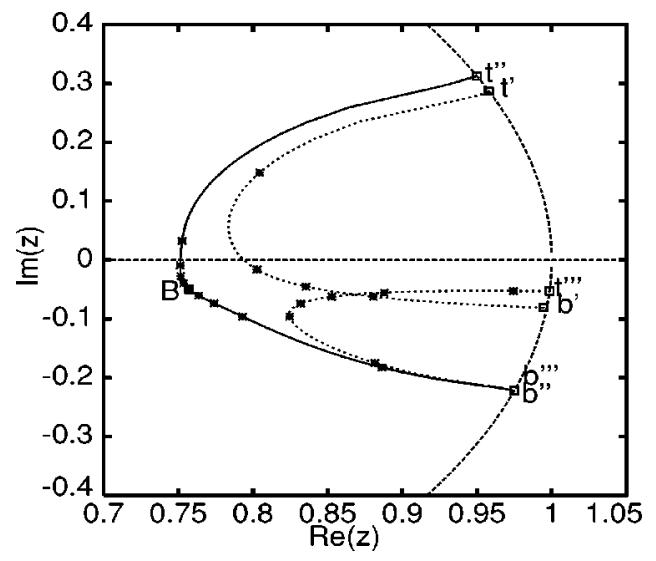

FIG. 4. Fixed-point trajectory when the energy is varied across the allowed band of the central cells. $e F=-3 \mathrm{meV} / \mathrm{nm}$. Dotted lines: first and third allowed bands of $M_{c}$. Solid line: second allowed band of $M_{c}$. The dashed line is the unit circle. The location of the fixed point at the Bragg energy is at the box labeled $B$.

seen, there is a sizable range of energies for which the fixed point describing the continuous path stays in the neighborhood of $B$, the Bragg energy. It is for this range of energies that the ARC design is efficient, because at the Bragg energy the fixed point is mapped by construction to the origin. And nearby points follow, because the mapping is conformal. For energies corresponding to the two dotted paths, transmission is also close to 1 due to a combination of the resonant transmission already present without the ARC cladding and the closeness of the leftmost parts of the paths to $B$. (Note that in the figure only a small part of the unit circle is shown.)

\section{B. Design and results for square barrier cells}

As a first example, we chose the parameters of our middle square barrier cell as those of the experiment of Pacher et al. ${ }^{5}$ The square barrier is of width $b_{m}=2.54 \mathrm{~nm}$ and height $V_{m}=290 \mathrm{meV}$. It is surrounded by half-wells of equal width, $w_{m} / 2$, with $w_{m}=6.50 \mathrm{~nm}$. The shape of the optimal filter is shown in the inset to Fig. 7. For simplicity in the numerical

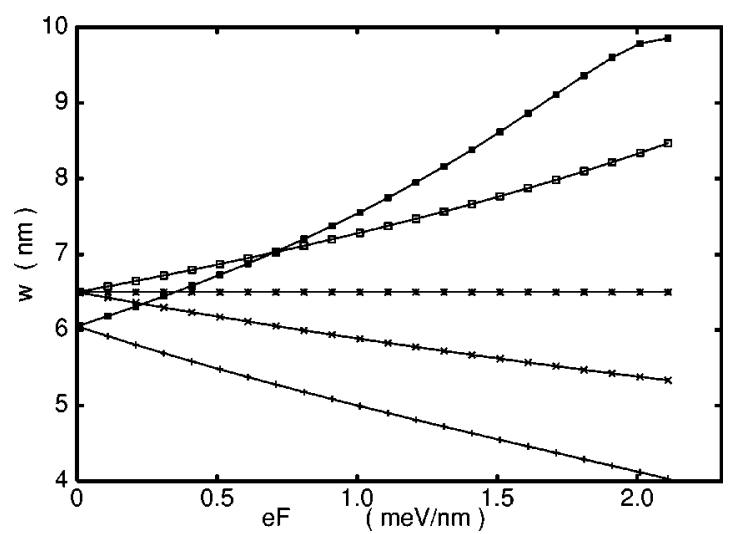

FIG. 5. Variation of the well width $w$ with applied electric field for square barrier cells, with optimal parameters of the ARC filter. From bottom to top on the right-hand side: $w_{a^{\prime}}(+), w_{l}(x), w_{m}$ (stars), $w_{r}$ (open boxes), $w_{a}$ (solid boxes).

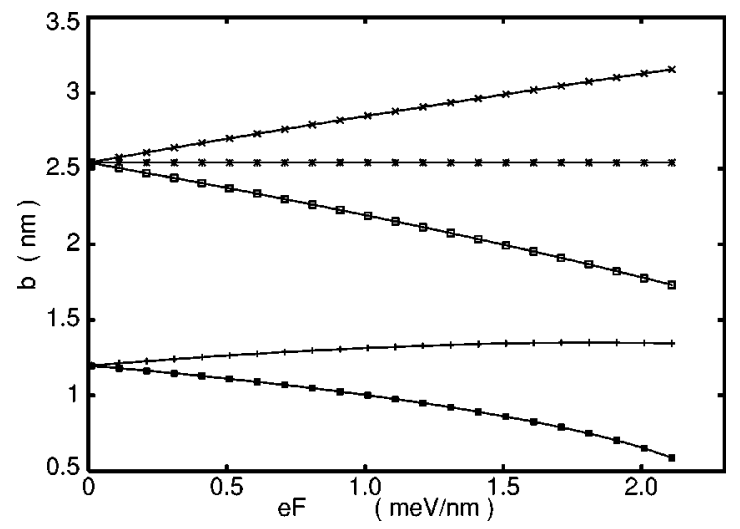

FIG. 6. Variation of the barrier width $b$ with applied electric field for square barrier cells, with optimal parameters of the ARC filter. From bottom to top in the right-hand side: $b_{a}$ (solid boxes), $b_{a^{\prime}}(+), b_{r}$ (open boxes), $b_{m}$ (stars), $b_{l}(x)$.

work we used in this example a common effective mass for barrier and well materials $m^{*} / m_{e}=0.071$ throughout. (This will be relaxed in the second example at the end of this section.) These choices place the lowest allowed band at $k_{b}$ $=0.3041 \mathrm{~nm}^{-1}<k<k_{t}=0.3693 \mathrm{~nm}^{-1}$, lower than in the previous example; see Fig. 1. This reduces the range of electric fields for which optimal filters can be found, due to the restriction already stated in Eqs. (22) and (23). To align the bands of the left cell, we impose Eqs. (16). To simplify the experimental implementation of the filters, we have also fixed the barrier height in every cell at $290 \mathrm{meV}$. This leaves two equations to determine the widths $w_{l}$ and $b_{l}$. To solve for them, we use a simplex method to minimize

$$
\mathcal{F}^{2} \equiv\left\{\operatorname{Re}\left[M_{l, 11}\left(E_{B}\right)\right]\right\}^{2}+\left\{\operatorname{Re}\left[M_{l, 11}\left(E_{t}\right)\right]+1\right\}^{2},
$$

with the matrix elements computed as described in the Appendix. Although the equations are nonlinear, this method has always produced a single physically useful set of $w_{l}, b_{l}$. We solve similar equations for the cell on the right. The resulting values are shown in Figs. 5 and 6; they vary smoothly with $e F$ in the range of electric fields explored.

The second step is the cladding with ARC cells. Let us consider first the rightmost ARC cell, subindex $a$. We again take $V_{a}=0.29 \mathrm{eV}$. The other two cell parameters $w_{a}$ and $b_{a}$ are determined by the condition that at the Bragg energy the ARC maps the origin to the fixed point of the three central cells, Eq. (14). We solve this equation by minimizing $\left|A_{21} / A_{11}-z_{f, i n}\right|$. Similarly we determine the parameters for the leftmost ARC cell by imposing Eq. (15). The parameters thus computed are included in Figs. 5 and 6. The resulting average transmissivities are shown in Fig. 7. The transmissivity remains close to the optimal value at zero field for most of the range explored. It drops sharply near the critical limit for a positive $e F$. This is seen in Fig. 8, where the transmission coefficients are for electric fields increasing from 0 to $2.1 \mathrm{meV} / \mathrm{nm}$ in steps of $0.3 \mathrm{meV} / \mathrm{nm}$.

Finally, for completeness, we have modified the example considered above by allowing different effective masses for barrier, $m_{b}^{*} / m_{e}=0.092$, and well, $m_{w}^{*} / m_{e}=0.067$, materials. Figure 9 shows transmission as a function of incident energy 


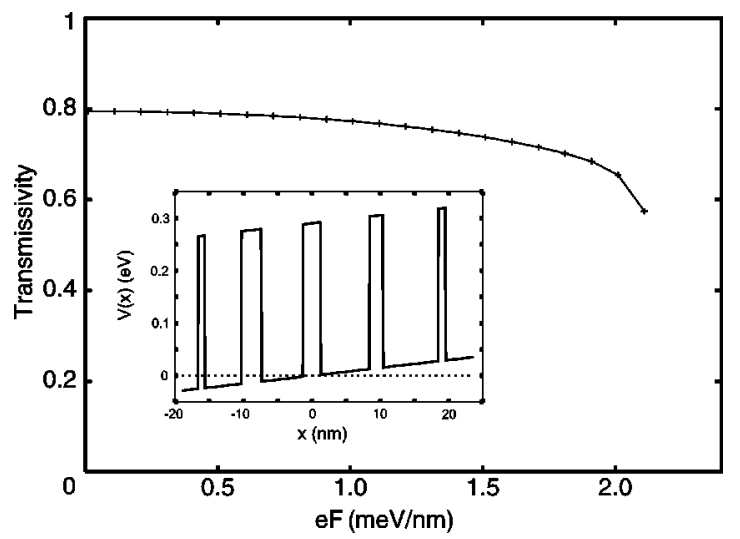

FIG. 7. Symbols + : transmissivities for the square barrier filters at the electric fields for which they are optimal. The solid line is drawn to guide the eye. Inset: shape of the optimal filter under applied bias of $1.5 \mathrm{mV} / \mathrm{nm}$.

for the optimal filters corresponding to $F=0$ and $F$ $=1.2 \mathrm{mV} / \mathrm{nm}$. The quality of the filters is similar to those of the previous example, with transmissivities of 0.80 and 0.75 for the two cases shown.

\section{SUMMARY AND CONCLUSIONS}

For a chosen electric field $F$, we have shown how to construct optimal band pass filters as finite superlattices. They are made of (i) an inner part, made of three cells with aligned bands, which define an energy window, and (ii) an ARC cladding consisting of a single cell on each side of the inner part. The design therefore is made in two stages: In the first, the values $w_{c}$ and $b_{c}$ for the central cell forming the inner part are chosen so as to define the desired energy window for the filter. This is done either using expressions already given in Ref. 1 or choosing the values from an experiment. ${ }^{5}$ Next the parameters of the left and right cells in that inner part, $w_{l, r}, b_{l, r}$, are determined imposing band alignment, via Eq. (25), and using the expressions for the transfer matrix given in the Appendix. With this parameter set we can then construct the total transfer matrix for the inner part for any energy and determine its fixed point $z_{f, i n}(E)$. In the second

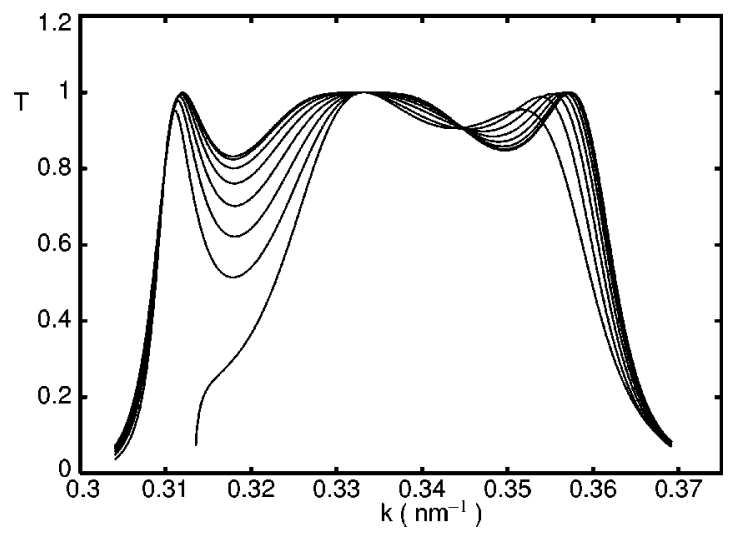

FIG. 8. Transmission $T(k)$ for the square barrier cell filters optimized for electric fields: $e F=0,0.3,0.6, \ldots, 2.1 \mathrm{meV} / \mathrm{nm}$.

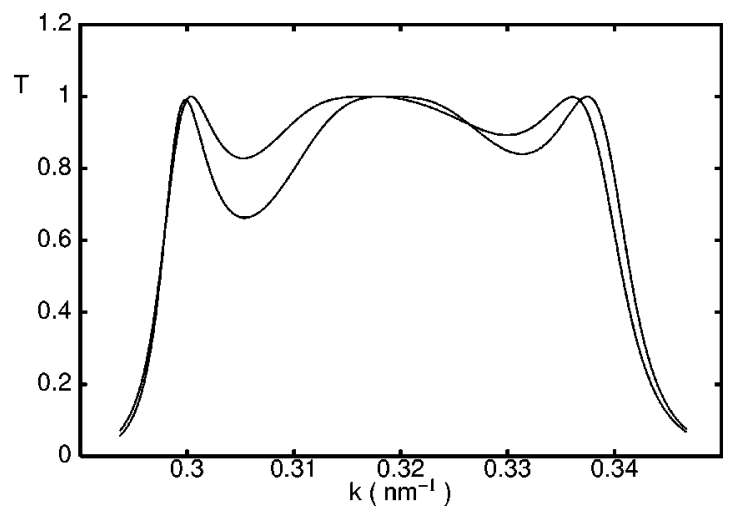

FIG. 9. Transmission $T(k)$ for the square barrier cell filters optimized for electric fields: $e F=0$ and $1.2 \mathrm{meV} / \mathrm{nm}$. Example with different effective masses for barrier and well materials.

stage, the parameters $w_{a, a^{\prime}}, b_{a, a^{\prime}}$ for the two ARC cells are determined imposing Eqs. (14) and (15). This is done at the Bragg energy, so as to guarantee unit transmission at this chosen value, located near the center of an allowed band. Following this method, we have shown that realistic values can be determined for the parameters of each of these cells and that the resulting filters have good transmissivities, provided the electric field of operation is within the range imposed by Eqs. (22) and (23).

We have found that for energies in the middle part of the transmission window the filter action is based on propagation of Bloch states, whereas for energies near the band boundaries part of the transmission is still resonant. We expect that the traversal times will be much longer at energies for which resonant transmission is significant. ${ }^{22}$ We have some preliminary numerical results, not shown here, confirming this and also that the ARC cladding always reduces those resonant times. We therefore expect that propagation in devices with an ARC filter will be less affected by phonon couplings, impurities, and other decoherence mechanisms. There should also be less of a buildup of space charge in the device that would affect the design. Work is in progress to obtain quantitative estimates for these effects.

\section{ACKNOWLEDGMENTS}

We are grateful to NSERC-Canada for Discovery Grant No. SAPIN-3198 (D.W.L.S., G.M.) and to DGES-Spain for continued support through Grant Nos. PB97-0915 and BFM2001-3710 (J.M.). This work was carried out as part of CERION-2, EU thematic network IST-2001-39059.

\section{APPENDIX: TRANSFER MATRIX FOR PIECEWISE LINEAR POTENTIALS}

We consider a square barrier cell extending from $x=x_{l}$ to $x=x_{r}$. The barrier is located between $x=x_{l, b}$ and $x=x_{r, b}$. We choose the origin of energy so that the potential is nil outside the barrier and $V_{b}$ inside, when $F=0$. The effective mass for the barrier is assumed to be energy independent and constant, $m_{b}^{*}$. For the rest of the cell and outside it, we take a constant value $m_{w}^{*}$. In addition there is a constant electric 
field $F$, directed along $x$, acting inside the cell. Furthermore, we assume that outside the cell the potential is constant and matches continuously to the cell values at the boundary $V(x)=e F x_{l}$ when $x<x_{l}$ and $V(x)=e F x_{r}$ when $x>x_{r}$. For convenience we write the wave function outside the cell, including explicit flux factors, as in Eq. (1), replacing $L, R$ by $l, r$. In each layer of constant effective mass, the wave function satisfies

$$
\begin{gathered}
-\frac{\hbar^{2}}{2 m_{b}^{*}} \phi^{\prime \prime}(x)+\left(e F x+V_{b}\right) \phi(x)=E \phi(x), \quad x_{l, b}<x<x_{r, b}, \\
-\frac{\hbar^{2}}{2 m_{w}^{*}} \phi^{\prime \prime}(x)+e F x \phi(x)=E \phi(x), \quad x_{l}<x<x_{l, b}, \\
x_{r, b}<x<x_{r} .
\end{gathered}
$$

Introducing the constants $\alpha_{w} \equiv\left(2 m_{w}^{*} e F / \hbar^{2}\right)^{1 / 3}, \quad \beta_{w} \equiv$ $-\alpha_{w} E /(e F)$ and the variable

$$
u=\alpha_{w} x+\beta_{w}
$$

the second equation in Eqs. (A1) simplifies to

$$
\phi_{u}^{\prime \prime}(u)-u \phi(u)=0 .
$$

This is the well-known Airy equation (Ref. 23, p. 446). It has two linearly independent solutions $\operatorname{Ai}(u)$ and $\operatorname{Bi}(u)$, whose Wronskian is $W(\operatorname{Ai}(u), \operatorname{Bi}(u))=1 / \pi$. For numerical convenience, we use a different pair of solutions

$$
\begin{gathered}
h^{(1)}(u) \equiv e^{-i \pi / 6} \sqrt{3}[\operatorname{Ai}(u)-i \operatorname{Bi}(u)]=\sqrt{-u} H_{1 / 3}^{(1)}(\xi), \\
h^{(2)}(u) \equiv e^{i \pi / 6} \sqrt{3}[\operatorname{Ai}(u)+i \operatorname{Bi}(u)]=\sqrt{-u} H_{1 / 3}^{(2)}(\xi),
\end{gathered}
$$

with

$$
\xi \equiv \frac{2}{3}(-u)^{3 / 2}
$$

The functions $H_{1 / 3}^{(1,2)}(\xi)$ are Hankel functions, as defined in Eq. 10.4.23 of Ref. 23. The Wronskian for this new pair is

$$
w \equiv W\left(h^{(2)}(u), h^{(1)}(u)\right)=-6 i / \pi .
$$

The solutions of the first of Eqs. (A1) can be determined similarly, but with $E$ replaced by $E-V_{b}$. This leads to new constants $\alpha_{b} \equiv\left(2 m_{b}^{*} e F / \hbar^{2}\right)^{1 / 3}$ and $\beta_{b} \equiv-\alpha_{b}\left(E-V_{b}\right) / e F$ and the variable

$$
v=\alpha_{b} x+\beta_{b} .
$$

The corresponding pair of linearly independent solutions is therefore $h^{(1)}(v)$ and $h^{(2)}(v)$. In terms of these dimensionless variables, we write the wave function as

$$
\begin{array}{rlr}
\phi(x)=a_{1} h^{(2)}(u)+b_{1} h^{(1)}(u) & \left(x_{l} \leqslant x<x_{l, b}\right) \\
=e_{1} h^{(2)}(v)+f_{1} h^{(1)}(v) & \left(x_{l, b} \leqslant x<x_{r, b}\right) \\
=c_{1} h^{(2)}(u)+d_{1} h^{(1)}(u) & \left(x_{r, b} \leqslant x<x_{r}\right) .
\end{array}
$$

By matching these solutions and their derivatives divided by the effective mass, at each boundary, we determine the transmission matrix $M$. To write the matching conditions in compact form, we define

$$
U(s) \equiv\left(\begin{array}{cc}
h^{(2)}(s) & h^{(1)}(s) \\
\frac{\alpha_{s}}{m_{s}} h^{(2)^{\prime}}(s) & \frac{\alpha_{s}}{m_{s}} h^{(1)^{\prime}}(s)
\end{array}\right),
$$

where $s=u$ or $v$ and the corresponding subindex indicates $w$ or $b$. The inverse is

$$
U^{-1}(s)=i \frac{\pi}{6}\left(\begin{array}{cc}
h^{(1)^{\prime}}(s) & -\frac{m_{s}}{\alpha_{s}} h^{(1)}(s) \\
-h^{(2)^{\prime}}(s) & \frac{m_{s}}{\alpha_{s}} h^{(2)}(s)
\end{array}\right) \text {. }
$$

With this notation, the matchings at $x=x_{l}, x=x_{l, b}, x$ $=x_{r, b}$, and $x=x_{r}$ give the following relations:

$$
\begin{aligned}
\frac{1}{\sqrt{\nu_{l}}}\left(\begin{array}{l}
a_{l} \\
b_{l}
\end{array}\right) & =\left(\begin{array}{cc}
1 / 2 & -i m_{w}^{*} /\left(2 k_{l}\right) \\
1 / 2 & i m_{w}^{*} /\left(2 k_{l}\right)
\end{array}\right) U\left(u_{l}\right)\left(\begin{array}{l}
a_{1} \\
b_{1}
\end{array}\right), \\
\left(\begin{array}{l}
a_{1} \\
b_{1}
\end{array}\right) & =U^{-1}\left(u_{l, b}\right) U\left(v_{l, b}\right)\left(\begin{array}{l}
e_{1} \\
f_{1}
\end{array}\right), \\
\left(\begin{array}{c}
e_{1} \\
f_{1}
\end{array}\right) & =U^{-1}\left(v_{r, b}\right) U\left(u_{r, b}\right)\left(\begin{array}{l}
c_{1} \\
d_{1}
\end{array}\right)
\end{aligned}
$$

$$
\sqrt{\nu_{r}}\left(\begin{array}{l}
c_{1} \\
d_{1}
\end{array}\right)=U^{-1}\left(u_{r}\right)\left(\begin{array}{cc}
1 & 1 \\
i k_{r} / m_{w}^{*} & -i k_{r} / m_{w}^{*}
\end{array}\right)\left(\begin{array}{l}
a_{r} \\
b_{r}
\end{array}\right) .
$$

Therefore,

$$
\begin{aligned}
M= & \sqrt{\frac{\nu_{l}}{\nu_{r}}}\left(\begin{array}{cc}
1 / 2 & -i m_{w}^{*} /\left(2 k_{l}\right) \\
1 / 2 & i m_{w}^{*} /\left(2 k_{l}\right)
\end{array}\right) U\left(u_{l}\right) U^{-1}\left(u_{l, b}\right) U\left(v_{l, b}\right) \\
& \times U^{-1}\left(v_{r, b}\right) U\left(u_{r, b}\right) U^{-1}\left(u_{r}\right)\left(\begin{array}{cc}
1 & 1 \\
i k_{r} / m_{w}^{*} & -i k_{r} / m_{w}^{*}
\end{array}\right),
\end{aligned}
$$

which is the desired result.

Tunneling under the barrier. Under the barrier, the values of $v$ may be large $(\simeq 20)$ and positive. This makes the Airy functions either negligible, $\operatorname{Ai}(v)$, or very large, $\operatorname{Bi}(v)$. Numerical calculations based on Eq. (A11) then become inaccurate. To mitigate this we perform some parts of the product $U\left(v_{l, b}\right) U^{-1}\left(v_{r, b}\right)$ analytically: 


$$
\begin{aligned}
P & \equiv U\left(v_{l, b}\right) U^{-1}\left(v_{r, b}\right)=i \frac{\pi}{6}\left(\begin{array}{cc}
h^{(2)}\left(v_{l, b}\right) & h^{(1)}\left(v_{l, b}\right) \\
\frac{\alpha_{b}}{m_{b}^{*}} h^{(2)^{\prime}}\left(v_{l, b}\right) & \frac{\alpha_{b}}{m_{b}^{*}} h^{(1)^{\prime}}\left(v_{l, b}\right)
\end{array}\right)\left(\begin{array}{cc}
h^{(1)^{\prime}}\left(v_{r, b}\right) & -\frac{m_{b}^{*}}{\alpha_{b}} h^{(1)}\left(v_{r, b}\right) \\
-h^{(2)^{\prime}}\left(v_{r, b}\right) & \frac{m_{b}^{*}}{\alpha_{b}} h^{(2)}\left(v_{r, b}\right)
\end{array}\right) \\
& =i \frac{\pi}{6}\left(\begin{array}{cc}
h_{l}^{(2)} h_{r}^{(1)^{\prime}}-h_{l}^{(1)} h_{r}^{(2))^{\prime}} & \frac{m_{b}^{*}}{\alpha_{b}}\left(-h_{l}^{(2)} h_{r}^{(1)}+h_{l}^{(1)} h_{r}^{(2)}\right) \\
\frac{\alpha_{b}}{m_{b}^{*}}\left(h_{l}^{(2)^{\prime}} h_{r}^{(1)^{\prime}}-h_{l}^{(1)^{\prime}} h_{r}^{(2)^{\prime}}\right) & -h_{l}^{(2)^{\prime}} h_{r}^{(1)}+h_{l}^{(1)^{\prime}} h_{r}^{(2)}
\end{array}\right),
\end{aligned}
$$

where in the third line we have simplified the notation in an obvious way. Remembering the definitions in Eqs. (A4) and that for large $v$ (see Ref. 23)

$$
\begin{gathered}
\operatorname{Ai}(v) \simeq \frac{1}{2 \sqrt{\pi}} v^{-1 / 4} e^{-2 / 3 v^{3 / 2}}, \\
\operatorname{Ai}^{\prime}(v) \simeq-\frac{1}{2 \sqrt{\pi}} v^{1 / 4} e^{-2 / 3 v^{3 / 2}}, \\
\operatorname{Bi}(v) \simeq \frac{1}{\sqrt{\pi}} v^{-1 / 4} e^{2 / 3 v^{3 / 2}}, \\
\operatorname{Bi}^{\prime}(v) \simeq \frac{1}{\sqrt{\pi}} v^{1 / 4} e^{2 / 3 v^{3 / 2}},
\end{gathered}
$$

we find

$$
\begin{aligned}
P_{11} & =\pi\left(\mathrm{Ai}_{l} \mathrm{Bi}_{r}^{\prime}-\mathrm{Bi}_{l} \mathrm{Ai}_{r}^{\prime}\right) \\
& \equiv \pi\left(e^{-2 / 3\left(v_{l}^{3 / 2}-v_{r}^{3 / 2}\right)} \widetilde{\mathrm{A}} i_{l} \widetilde{\mathrm{B}} i_{r}^{\prime}-e^{2 / 3\left(v_{l}^{3 / 2}-v_{r}^{3 / 2}\right)} \widetilde{\mathrm{B}} i_{l} \widetilde{\mathrm{A}} i_{r}^{\prime}\right),
\end{aligned}
$$

where, guided by the asymptotic expressions, we have defined

$$
\begin{gathered}
\widetilde{\mathrm{A}} \equiv e^{2 / 3 v^{3 / 2}} \mathrm{Ai}(v), \quad \widetilde{\mathrm{A}} \mathrm{i}^{\prime} \equiv e^{2 / 3 v^{3 / 2}} \mathrm{Ai}^{\prime}(v), \\
\widetilde{\mathrm{B}} i \equiv e^{-2 / 3 v^{3 / 2}} \mathrm{Bi}(v), \quad \widetilde{\mathrm{B}} i^{\prime} \equiv e^{-2 / 3 v^{3 / 2}} \operatorname{Bi}^{\prime}(v) .
\end{gathered}
$$

The advantage of Eq. (A14) is that now the exponential factors that made the Ai negligibly small and the Bi very large are compensated leaving the remaining expressions well behaved. $\widetilde{A} i$ and $\widetilde{B} i$ are computed using the full asymptotic series. $^{23}$

The other matrix elements of $P$ are handled similarly, giving (with $\lambda=e^{-2 / 3\left(v_{r}^{3 / 2}-v_{l}^{3 / 2}\right)}$ )

$$
\begin{gathered}
P_{12}=\pi\left(\lambda \widetilde{\mathrm{B}} i_{l} \widetilde{\mathrm{A}} i_{r}-\lambda^{-1} \widetilde{\mathrm{A}} i_{l} \widetilde{\mathrm{B}} i_{r}\right) \frac{m_{b}^{*}}{\alpha_{b}}, \\
P_{21}=\pi\left(\lambda \widetilde{\mathrm{B}} i_{l}^{\prime} \widetilde{\mathrm{A}} i_{r}^{\prime}-\lambda^{-1} \widetilde{\mathrm{A}} i_{l}^{\prime} \widetilde{\mathrm{B}} i_{r}^{\prime}\right) \frac{\alpha_{b}}{m_{b}^{*}}, \\
P_{22}=\pi\left(\lambda \widetilde{\mathrm{A}} i_{r} \widetilde{\mathrm{B}} i_{l}^{\prime}-\lambda^{-1} \widetilde{\mathrm{B}} i_{r} \widetilde{\mathrm{A}} i_{l}^{\prime}\right) .
\end{gathered}
$$

\section{M matrix for a $\delta$ cell}

When the barrier has zero width, $V_{\delta}(x)=C \delta\left(x-x_{b}\right)$, the expression for the $M$ matrix simplifies. Taking into account that the wave function is continuous at $x_{b}$ while the first derivative has a jump, one finds

$$
\lim _{\epsilon \rightarrow 0} \int_{u_{b}-\epsilon}^{u_{b}+\epsilon} \frac{d^{2} \phi(u)}{d u^{2}} d u=\gamma \phi\left(u_{b}\right), \quad \gamma=\frac{2 m^{*}}{\hbar^{2}} \frac{C}{|\alpha|} .
$$

The matching condition at $x_{b}$ implies

$$
\begin{aligned}
U\left(u_{b}\right)\left(\begin{array}{l}
a_{1} \\
b_{1}
\end{array}\right)= & \left(\begin{array}{cc}
h^{(2)}\left(u_{b}\right) & h^{(1)}\left(u_{b}\right) \\
h^{(2)^{\prime}}\left(u_{b}\right)-\gamma h^{(2)}\left(u_{b}\right) h^{(1)^{\prime}}\left(u_{b}\right)-\gamma h^{(1)}\left(u_{b}\right)
\end{array}\right) \\
& \times\left(\begin{array}{l}
c_{1} \\
d_{1}
\end{array}\right) .
\end{aligned}
$$

Inverting, we get

$$
\begin{aligned}
& \left(\begin{array}{cc}
1+\gamma h^{(1)}\left(u_{b}\right) h^{(2)}\left(u_{b}\right) / w & \gamma\left(h^{(1)}\left(u_{b}\right)\right)^{2} / w \\
-\gamma\left(h^{(2)}\left(u_{b}\right)\right)^{2} / w & 1-\gamma h^{(1)}\left(u_{b}\right) h^{(2)}\left(u_{b}\right) / w
\end{array}\right)\left(\begin{array}{l}
c_{1} \\
d_{1}
\end{array}\right) \\
& =\left(\begin{array}{l}
a_{1} \\
b_{1}
\end{array}\right),
\end{aligned}
$$

which replaces the second and third equations in Eqs. (A10). In the above, $w$ denotes the Wronskian. 
${ }^{1}$ G.V. Morozov, D.W.L. Sprung, and J. Martorell, J. Phys. D 35, 2091 (2002).

${ }^{2}$ G.V. Morozov, D.W.L. Sprung, and J. Martorell, J. Phys. D 35, 3052 (2002).

${ }^{3}$ D.W.L. Sprung, G.V. Morozov, and J. Martorell, J. Appl. Phys. 93, 4395 (2003).

${ }^{4}$ C. Pacher, C. Rauch, G. Strasser, E. Gornik, F. Elsholz, A. Wacker, G. Kiesslich, and E. Schöll, Appl. Phys. Lett. 79, 1486 (2001).

${ }^{5}$ C. Pacher, G. Strasser, E. Gornik, F. Elsholz, G. Kiesslich, A. Wacker, and E. Schöll, Physica E 12, 285 (2002).

${ }^{6}$ M. Kast, C. Pacher, G. Strasser, E. Gornik, and W.S.M. Werner, Phys. Rev. Lett. 89, 136803 (2002).

${ }^{7}$ C. Gmachl, F. Capasso, D.L. Sivco, and A.Y. Cho, Rep. Prog. Phys. 64, 1533 (2001).

${ }^{8}$ C. Rauch, G. Strasser, K. Unterrainer, W. Boxleitner, and E. Gornik, Phys. Rev. Lett. 81, 3495 (1998).

${ }^{9}$ T.K. Gaylord, E.N. Glytsis, and K.F. Brennan, J. Appl. Phys. 65, 2535 (1989).

${ }^{10}$ E.N. Glytsis, T.K. Gaylord, and K.F. Brennan, J. Appl. Phys. 66, 1494 (1989).

${ }^{11}$ T.K. Gaylord, E.N. Glytsis, and K.F. Brennan, J. Appl. Phys. 67, 2623 (1990).
${ }^{12}$ C.C. Chang and C.S. Kuo, J. Phys. D 32, 139 (1999).

${ }^{13}$ H.H. Tung and C.P. Lee, IEEE J. Quantum Electron. 32, 507 (1996).

${ }^{14}$ H.H. Tung and C.P. Lee, IEEE J. Quantum Electron. 32, 2122 (1996).

${ }^{15}$ I. Gomez, F. Dominguez-Adame, E. Diez, and V. Bellani, J. Appl. Phys. 85, 3916 (1999).

${ }^{16}$ H.P. Simanjuntak and P. Pereyra, Phys. Rev. B 67, 045301 (2003).

${ }^{17}$ D.F. Nelson, R.C. Miller, and D.A. Kleinman, Phys. Rev. B 35, 7770 (1987).

${ }^{18}$ T. Yonte, J.J. Monzón, L.L. Sánchez-Soto, J.F. Cariñena, and C. López-Lacasta, J. Opt. Soc. Am. A 19, 603 (2002); see also physics/0104050 (unpublished).

${ }^{19}$ J.J. Monzón, T. Yonte, L.L. Sánchez-Soto, and J.F. Cariñena, J. Opt. Soc. Am. A 19, 985 (2002); see also physics/0202053 (unpublished).

${ }^{20}$ D.W.L. Sprung, G.V. Morozov, and J. Martorell, J. Phys. A 37, 1861 (2004).

${ }^{21}$ D.W.L. Sprung, Hua Wu, and J. Martorell, Am. J. Phys. 61, 1118 (1993).

${ }^{22}$ R. Romo, Phys. Rev. B 66, 245311 (2002).

${ }^{23}$ Handbook of Mathematical Functions, edited by M. Abramowitz and I.A. Stegun (Dover, New York, 1970). 